\title{
Surprises from a deep ASCA spectrum of a Broad Absorption Line Quasar PHL 5200
}

\author{
S. Mathur ${ }^{1,2}$, G. Matt ${ }^{3}$, P. J. Green², M. Elvis ${ }^{2}$, K. P. Singh ${ }^{4}$
}

\begin{abstract}
We present a deep ( $\sim 55 \mathrm{ksec})$ ASCA observation of the prototype Broad Absorption Line Quasar (BALQSO) PHL 5200. This is the best X-ray spectrum of a BALQSO yet. We find that (1) the source is not intrinsically X-ray weak, (2) the line of sight absorption is very strong with $\mathrm{N}_{\mathrm{H}}=5 \times 10^{23} \mathrm{~cm}^{-2}$, (3) the absorber does not cover the source completely; the covering fraction is $\approx 90 \%$. This is consistent with the large optical polarization observed in this source, implying multiple lines of sight. The most surprising result of this observation is that (4) the spectrum of this BALQSO is not exactly similar to other radio-quiet quasars. The hard X-ray spectrum of PHL 5200 is steep with the power-law spectral index $\alpha \approx 1.5$. This is similar to the steepest hard X-ray slopes observed so far. At low redshifts, such steep slopes are observed in narrow line Seyfert 1 galaxies, believed to be accreting at a high Eddington rate. This observation strengthens the analogy between BALQSOs and NLS1 galaxies and supports the hypothesis that BALQSOs represent an early evolutionary state of quasars (Mathur 2000). It is well accepted that the orientation to the line of sight determines the appearance of a quasar; age seems to play a significant role as well.

Subject headings: galaxies: active - quasars: absorption lines-quasars: individual (PHL 5200)—X-rays: galaxies
\end{abstract}

\section{Introduction}

Broad absorption line quasars, in which the kinetic energy carried out in the absorbing outflow is a significant fraction of the bolometric luminosity of the quasar, offer a challenge to our understanding of the quasar energy budget (as suggested in Mathur, Elvis \& Wilkes 1995; see also $\S 11.3$ in Krolik 1999). At the same time, they also offer new insights into the nuclear structure of quasars (Ogle 1998, Elvis 2001). X-ray observations are important in this investigation as they offer precise measurements of absorbing column densities. Combined UV and X-ray analysis is a powerful tool to understand the physical con-

\footnotetext{
${ }^{1}$ The Ohio State Univ., smita@astronomy.ohiostate.edu

${ }^{2}$ Harvard Smithsonian Center for Astrophysics

${ }^{3}$ Universitá Roma Tre, Italy

${ }^{4}$ Tata Institute of Fundamental Research, Mumbai, India
}

ditions in the absorbing gas (Mathur et al. 1998 and references there in). However, X-ray observations of BALQSOs have essentially resulted in non-detections (Green \& Mathur 1996). While the available evidence clearly suggested that the observed X-ray weakness of BALQSOs is a result of strong X-ray absorption (Mathur et al. 2000 and references there in), in some objects absorption was not apparent (Gallagher et al. 1999). So the nature of X-ray weakness of BALQSOs and the column density of absorption if any, could not be determined with certainty for the lack of a good X-ray spectrum.

PHL 5200, the prototype BALQSO at $\mathrm{z}=1.98$ (Burbidge 1968) was clearly detected in EXOSAT medium energy experiment (ME), but not by the low energy experiment (LE) (Singh, Westergaard \& Schnopper 1987). To obtain consistency between ME and LE requires a column density of $\gtrsim 10^{22} \mathrm{~cm}^{-2}$ at the source. The detection of PHL 5200 with EXOSAT made it an excellent can- 
didate for detailed spectral study in X-rays. A 1994 ASCA observation of PHL 5200 yielded the first X-ray spectrum of a BALQSO (Mathur, Elvis \& Singh 1995, MES95 here after), and remained the only one until this year. However, the quality of the ASCA spectrum was poor and the parameters of the fit were not well determined in the 17.7 ksec observation. The photon index of the best-fit power-law was uncertain to \pm 0.9 (all the errors are quoted to $90 \%$ confidence, unless noted otherwise). While excess absorption with $\mathrm{N}_{\mathrm{H}}=1.3_{-1.1}^{+2.3} \times 10^{23}$ $\mathrm{cm}^{-2}$ at the source provided a better fit, a model with only Galactic absorption was acceptable (see also Gallagher et al. 1999). A good signal to noise spectrum of a BALQSO was very much needed and PHL 5200 remained the best target. So we reobserved PHL 5200 with ASCA, to obtain better quality data and better constrain the parameters of an X-ray spectrum of a BALQSO. We find that the spectrum contains more surprises that affect the interpretation of the BAL phenomenon.

\section{Observations and Data Analysis}

\subsection{Observations}

We observed PHL 5200 with ASCA (Tanaka et al. 1994) on 1999 November 21. ASCA contains two sets of two detectors, SIS (Solid-state Imaging Spectrometer) and GIS (Gas Imaging Spectrometer). The effective exposure times in SIS0, SIS1, GIS2 and GIS3 were 83,522 seconds, 73,320 seconds, 93,385 seconds and 93,337 seconds respectively. The SIS was operated in 1CCD mode with the target in the standard 1CCD mode position. The GIS was operated in pulse height $(\mathrm{PH})$ mode. The data were reduced and analyzed using FTOOLS and XSELECT in a standard manner (see ASCA Data Reduction Guide for details of data reduction).

\subsection{Data Analysis}

The source was clearly detected in all the four instruments with background subtracted net count rate of $8.6 \pm 0.5 \times 10^{-3}, 4.8 \pm 0.4 \times 10^{-3}$, $5.6 \pm 0.6 \times 10^{-3}, 10.9 \pm 0.6 \times 10^{-3}$ for SIS0, SIS1, GIS2 and GIS3 respectively. We extracted the source spectrum from a circular region centered on the source. For GIS2 and GIS3 we used the recommended $6^{\prime}$ radius. For SIS0 and SIS1 we used $3^{\prime}$ and $2^{\prime}$ radii, respectively. Larger regions were not possible as the source was quite close to the chip gap. Background events were extracted from the the identical regions for each instruments from the deep background images supplied by the ASCA guest observed facility. For GIS, the latest (March 1995) response matrices were used, while for SIS, they were generated using FTOOL sisrmg. The spectra were then corrected for vignetting and the channels were grouped to contain at least 15 net counts per channel. In summary, the spectra for all instruments were extracted in a standard manner.

We also extracted background from different source-free regions on the same detector from our PHL 5200 observation. We found that the spectra extracted in this way did not alter our results in any significant way. For the rest of the paper we will discuss only the spectra with background extracted from the deep background observations.

Our observations were taken towards the end of ASCA mission, and the CCDs on the satellite were significantly degraded over time. There are problems with the charge transfer inefficiency (CTI), the residual dark distribution (RDD), and an unidentified additional loss of SIS low energy efficiency. ${ }^{5}$ For our observation in the 1 CCD mode and for a weak source like PHL 5200, the latter is the most serious issue. The loss of the low energy response of the two SIS detectors can be parameterized with excess absorption (Yaqoob et al. 2000). This " $\mathrm{N}_{\mathrm{H}}$ correction" is different for SIS0 and SIS1 and is a function of time. We have taken into account all the calibration uncertainties carefully in our spectral extraction and analysis.

\subsection{Spectral Analysis}

The extracted spectra were analyzed using XSPEC version 11.0 (Arnaud, 1996). Data below $0.6 \mathrm{keV}$ for SIS, below $0.9 \mathrm{keV}$ for GIS and above $9.5 \mathrm{keV}$ for all the instruments were ignored in spectral fits as these energy channels are not well calibrated. We first fitted the spectra from each instrument separately and found the results to be generally consistent. We then fitted all the spectra simultaneously leaving the normalizations free, to allow for small differences in absolute calibration.

As a first step we fitted the spectra with a

\footnotetext{
${ }^{5}$ For ASCA Calibration Uncertainties see: http://legacy.gsfc.nasa.gov/docs/asca/cal_probs.html
} 
simple power-law and Galactic absorption (with $\mathrm{N}_{\mathrm{H}}=4.8 \times 10^{20} \mathrm{~cm}^{-2}$, Stark et al. 1992). The " $\mathrm{N}_{\mathrm{H}}$ correction" discussed in section 2 was applied to the SIS spectra, which for our epoch of observation was $6.8 \times 10^{20} \mathrm{~cm}^{-2}$ for SIS0 and $9.8 \times 10^{20}$ $\mathrm{cm}^{-2}$ for SIS1, with $\approx \pm 10 \%$ uncertainty. The fit was not good, with $\chi^{2}=226.5$ for 137 degrees of freedom. As excess absorption was indicated in the earlier spectrum of PHL 5200 (MES95) and in other BALQSOs (Mathur et al. 2000 and references there in), absorption at the source was added as a next step. The fit was better, but clearly showed negative residuals around one $\mathrm{keV}$, and positive residuals at lower energies (Fig. 1, Fig. 2). Note that the behavior of the low energy residuals is exactly opposite to that due to the low energy calibration problem which produces spurious negative residuals. The implied recovery of the underlying power-law is a typical signature of partial covering by the absorber. So we fitted the spectrum with a partial covering model and the fit was significantly better $\left(\chi^{2}=182.5,135\right.$ degrees of freedom). The resulting $\mathrm{N}_{\mathrm{H}}=5 \pm 1 \times 10^{23}$ $\mathrm{cm}^{-2}$ and covering factor is $0.9_{-0.06}^{+0.05}$. The confidence contours of the interesting parameters are plotted in figure 3 . The scale over which figure 3 is plotted shows the range of $3 \sigma$ uncertainty in the earlier observation (MES95). The column density at the source is greater than $2 \times 10^{23} \mathrm{~cm}^{-2}$ and the power-law slope $\alpha$ is greater than 1.2 at $99 \%$ confidence (flux $f(E) \propto E^{-\alpha}$ where $\mathrm{E}$ is the energy).

The only residuals left after the partial covering model fit, are those above $\sim 8 \mathrm{keV}$ (observed frame), lying below the model values (Figure 2). Such residuals are not expected to be due to any calibration problems. They imply that a simple power-law continuum must turn over at higher energies. So we added a high energy cutoff to the model and re-fitted the data. This resulted in a much better fit with $\chi_{\nu}^{2}=1.18$. This "best fit" model, however, resulted in an unusually low value of the high energy cut-off of $\mathrm{E}_{\text {cuoff }}=18$ $\mathrm{keV}$ (rest-frame) with e-folding energy of $0.4 \mathrm{eV}$. The typical high energy cutoff in quasar spectra is believed to be about $300 \mathrm{keV}$, though Yaqoob (2000) has drawn attention to the fact that this value is not well measured. We are aware of at least one another AGN with a very low cutoff value. In ESO 103-G35, Wilkes et al. (2001) found
$\mathrm{E}_{\text {cutoff }}=29 \pm 10 \mathrm{keV}$. Low values of $\mathrm{E}_{\text {cuoff }}$ are likely to be important in understanding the spectrum of the cosmic X-ray background (Yaqoob, 2000). We note, however, that the negative residuals observed in our spectrum of PHL 5200 are significant only in the last few channels. So we caution against attaching too much significance to this result.

As discussed above, the source is highly absorbed with $\mathrm{N}_{\mathrm{H}}=5 \times 10^{23} \mathrm{~cm}^{-2}$. While the absorber is not Thomson thick, the effects of Thomson and Compton scattering may start becoming important at such column densities. In the analysis above, only photoelectric absorption is taken into account. The negative residuals observed at higher energies could be the effect of excess absorption due to Compton scattering, though for the observed column density it is not expected to be a significant effect (Matt et al. 1999). Nevertheless, we re-fitted our spectra with a partial covering model that fully incorporates Compton scattering in the absorber, as discussed in Matt et al. (1999). The resulting parameters are: $\mathrm{N}_{\mathrm{H}}=6 \pm 2 \times 10^{23} \mathrm{~cm}^{-2}, \alpha=1.9 \pm 0.4$, and covering fraction $=0.94 \pm 0.04$. These are consistent with the model without Compton scattering, as expected.

For a spherical distribution of matter with $\mathrm{N}_{\mathrm{H}}=5 \times 10^{23} \mathrm{~cm}^{-2}$, a Fe-K emission line with equivalent width (EW) of $270 \mathrm{eV}$ is expected (as measured against the observed continuum). We do not detect any line at the position of $\mathrm{Fe}-\mathrm{K} \alpha$ (rest energy $6.4 \mathrm{keV}$ ). The upper limit on the observed $\mathrm{EW}$ for a narrow line is $71 \mathrm{eV}$ for SIS0 data and $102 \mathrm{eV}$ for GIS3 data. The corresponding restframe EWs are $212 \mathrm{eV}$ and $304 \mathrm{eV}$ respectively. So we cannot put any meaningful constraint on the geometry of the absorber as viewed by the continuum source.

\section{Discussion}

The BALQSO PHL 5200 is clearly detected in all the instruments in this deep ASCA observation. With a total of 2610 "good" counts, this is the best X-ray spectrum of a BALQSO yet. The parameters of the spectral fit are well constrained; the X-ray flux is highly absorbed with $\mathrm{N}_{\mathrm{H}}=5 \pm 1 \times 10^{23} \mathrm{~cm}^{-2}$. While consistent with the conclusions of MES95, the earlier low signal to 
noise spectrum was also consistent with a model with no excess absorption (see also Gallagher et al. 1999). We unambiguously confirm large absorbing column density in PHL 5200. The X-ray weakness of BALQSOs thus is surely a result of absorption.

The absorber does not cover the continuum fully, with a covering fraction $90 \pm 5 \%$. This must be the reason for the apparent lack of excess absorption and flatter spectrum in the low signal to noise spectrum (see also Green et al. 2001). Partial covering of the absorber is consistent with the large optical polarization observed in this source (Goodrich \& Miller 1995, Cohen et al. 1995) implying that there are at least two lines of sight to the nucleus: one direct, highly absorbed, and another scattered but unabsorbed. Gallagher et al. (1999) have discussed the possibility that partial covering by the absorber might be responsible for highly polarized BALQSOs to be relatively Xray bright. Here we find that indeed, that is the best fit model to the PHL 5200 spectrum.

An associated absorber with high column density and partially covering the continuum is our preferred interpretation of the observed spectrum of PHL 5200. While physically motivated, this is not the only way to model the ASCA spectrum. For example, the low energy recovery of the absorbed spectrum may be be modeled as an unrelated soft-excess. ${ }^{6}$ The $\operatorname{dip}$ at $\approx 1 \mathrm{keV}$ is also a signature of a warm absorber. Note, however, that in PHL 5200 it corresponds to rest-frame energy of $\approx 3 \mathrm{keV}$. Since absorption due to highly ionized sulphur or silicon occurs at these energies, such an interpretation might be plausible. However, it seems unlikely given that these are not among the most abundant elements.

The most surprising result of this observation is the steep spectrum, with X-ray power-law slope $\alpha=1.7 \pm 0.4$. This might be an additional reason behind the unexpected non-detections of BALQSOs even in sensitive hard X-ray observations $(\S 1)$. The mean ASCA slope for radio-quiet quasars at high redshifts is $\alpha=0.67 \pm 0.11$, with a dispersion of $\sigma=0.07$ (Vignali et al. 1999, in the reshift

${ }^{6}$ The nearby object, $\approx 8^{\prime \prime}$ away from the optical position of PHL 5200, is an early K star with no emission lines, and is therefore very unlikely to contribute to the X-ray flux of PHL 5200. interval between $\mathrm{z}=1.9$ and 2.3). Our observations imply that the spectra of BALQSOs may not be exactly like other radio-quiet quasars, but are steeper. The observed, absorption corrected, 2-10 $\mathrm{keV}$ flux is $3.7 \times 10^{-13} \mathrm{ergs} \mathrm{cm}^{-2} \mathrm{~s}^{-1}$ (SIS0), consistent with the 1994 ASCA observation (MES95). So, the observed steep spectrum is unlikely to be a result of a short lived high-state. The only other BALQSO, PG 2112+059, for which a spectrum is available, has $\alpha=0.98_{-0.27}^{+0.4}$ (Gallagher et al. 2001). As noted by Gallagher et al. , this is consistent with the mean quasar slope. However, it is also consistent with as steep as 1.38 at $90 \%$ confidence.

As discussed in section 2.2, CCDs on ASCA have been degraded over time. While we have been very careful in our analysis, there might be some unknown calibration uncertainties which have affected the results presented here. Future observations with Chandra and XMM-Newton will be useful in this respect. The discussion below assumes that these ASCA results are correct.

At low redshifts, Brandt, Mathur \& Elvis (1997) found that the hard X-ray spectra of Seyfert galaxies are typically flatter than about $\alpha=1.0$. The Seyfert galaxies with steeper spectra are the narrow line Seyfert 1 galaxies (NLS1s). Brandt (2000) has discussed the analogy between low ionization BALQSOs and NLS1 galaxies. Mathur (2000) has discussed the analogy between BALQSOs and NLS1s further and has argued that NLS1 may be active galactic nuclei (AGNs) in the making. In this scenario, young radio-quiet AGNs are accreting at a high Eddington rate and have steep spectra. Over time, the accretion rate drops and the X-ray spectrum flattens. BALQSOs may be in that early evolutionary phase when the shroud surrounding the nuclear black-hole is being blown away and a quasar emerges (e.g. Fabian 1999, see also Becker et al. 2000). If the observed steep spectrum of PHL 5200 is a general property of BALQSOs, then it supports the evolutionary hypothesis of Mathur (2000) and further supports their analogy with NLS1 galaxies. On the other hand, PHL 5200 is one of the X-ray brightest BALQSOs, and so may not be representative of the BALQSO population. What is more likely, however, is that its orientation and cloud geometry fortuitously allow for substantial reflection of X-rays into our line of sight. 
It has been an accepted paradigm that the orientation to the line of sight determines the appearance of an AGN. In addition to orientation, the age of a quasar also seems to play a role in its appearance. The steep X-ray spectra in NLS1s are a result of their high accretion rate, close to the Eddington limit (Pounds et al. 1995). BALQSOs, however, are much more luminous than NLS1s, and so must have far more massive black holes. Steep X-ray spectra in such systems may prove to be challenging to accretion theories.

We thank Tahir Yaqoob for help with ASCA calibration uncertainties, and Perry Berlind for obtaining the optical spectrum of the $\mathrm{K}$ star at the Fred Lawrence Whipple Observatory. This work is supported in part by NASA grants NAG5-9270 \& NAG5-8913 (LTSA) to SM.

\section{REFERENCES}

Arnaud, K. A. 1996 in ASP Conf. Ser. 101 Astronomical Data Analysis Software and Systems V, ed. G. Jacoby \& J. Barnes (San Fracisco: ASP), 17

Becker, R., White, R., Gregg, M., Brotherton, M., Laurent-Muehleisen, S., Arav, N., 2000, ApJ, 538,72

Burbidge, E. M. 1968, ApJ, 152, 111

Brandt, W. N. 2000, in New Astronomy Reviews, 4, 461

Brandt, W. N., Mathur, S., \& Elvis, M., 1997, MNRAS, 285, L25

Cohen et al. 1995, ApJL, 448,77

Elvis, M. 2001, ApJ, in press.

Fabian, A.C. 1999, 308, 39

Gallagher, S., Brandt, W. N., Sambruna, R., Mathur, S., \& Yamasaki, N. 1999, ApJ, 519, 549

Gallagher, S., Brandt, W. N., Laor, A., Elvis, M., Mathur, S., Wills, B. J. 2001, ApJ, in press.

Goodrich, R. \& Miller, 1995, ApJ, 448, 73L

Green, P. J. \& Mathur, S. 1996 ApJ, 462, 637
Green, P. J., Aldcroft, T., \& Mathur, S. 2001, in preparation.

Krolik, J. 1999, "Active Galactic Nuclei" (Princeton University Press)

Mathur, S. 2000, MNRAS Letters, 314, L17

Mathur, S., Elvis, M., \& Singh, K. P. 1995, ApJ, 455, L9 (MES95)

Mathur, S., Elvis, M., \& Wilkes, B. 1995, ApJ, 452,230

Mathur, S., Wilkes, B., \& Elvis, M. 1998, ApJL, 503, L23

Mathur, S. et al. 2000, ApJL, 533, L79

Matt, G., Pompilio, F., \& La Franca, F. 1999, New Astronomy, 4, 191

Pounds, K., Done, C., \& Osborn, J. 1995, MNRAS, 277, L5

Ogle, P. 1998, Ph.D. Thesis, California Institute of Technology.

Singh, K. P., Westergaard, N. J., \& Schnopper, H. W., 1987, A \& A, 172, L11

Tanaka, Y., Holt, S.S., \& Inoue, H. 1994, PASJ, 46, L37

Vignali, C., Comastri, A., Cappi, M., Palumba, G., Matsuoka, M., \& Kubo, H., 1999, ApJ, 516, 582

Wilkes, B.J., Mathur, S., Fiore, F., Antonelli, A., Nacastro, F., 2001, ApJ, in press

Yaqoob, T. 2000, in 'Large Scale Structure in the X-ray Universe" , Ed: M. Plionis and I. Georgantopoulos (Editions Frontieres), p. 257

This 2-column preprint was prepared with the AAS LATEX macros v5.0. 
Table 1: Spectral fits to ASCA data of PHL5200

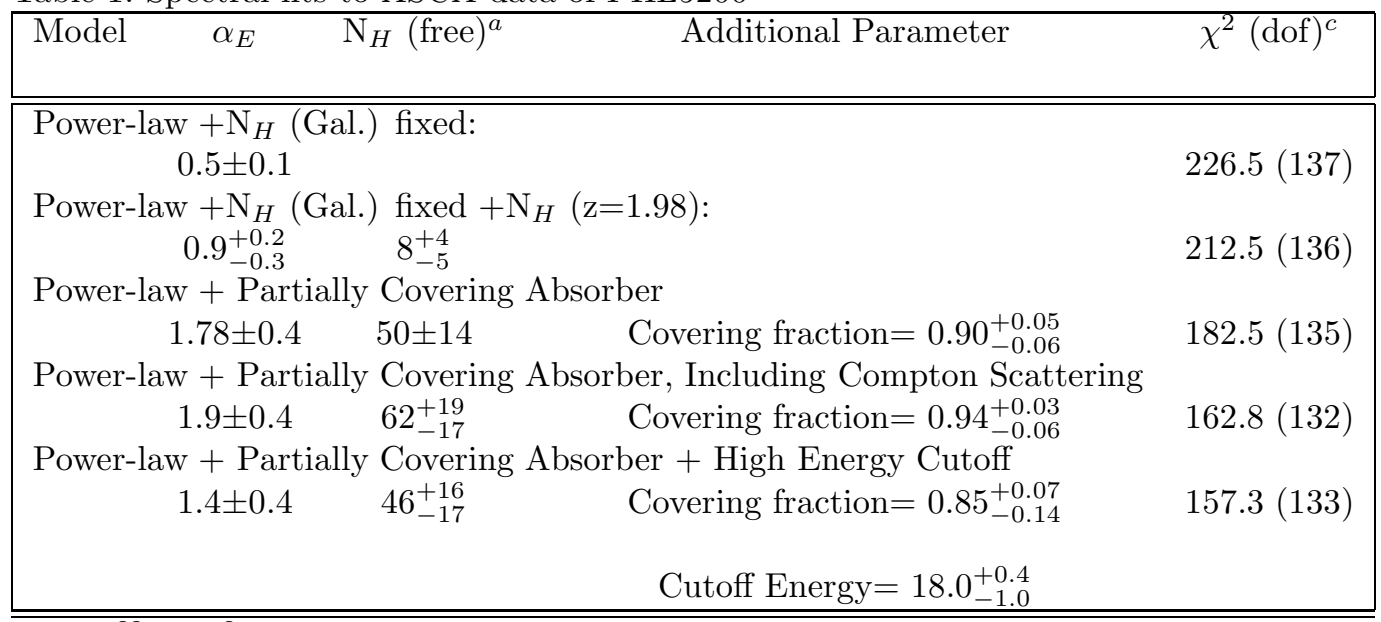

a: $\times 10^{22} \mathrm{~cm}^{-2}$

b: in units of $10^{-4}$ photons $\mathrm{keV}^{-1} \mathrm{~cm}^{-2} \mathrm{~s}^{-1}$ at 1 $\mathrm{keV}$

c: degrees of freedom. 
Fig. 1.- Residuals $\left(\Delta \chi^{2}\right)$ to a power-law fit showing clear signature of a partially covering absorber. Here the power-law was fitted only to the data above $2 \mathrm{keV}$ and extrapolated to lower energies. Note the strong turn-over below $2 \mathrm{keV}$ and recovery below about $1 \mathrm{keV}$. Only SIS data are shown for clarity; dots: SIS0, triangles: SIS1.

Fig. 2.- ASCA data of PHL 5200 divided by fitted models: (from top to bottom) (a) power-law, Galactic absorption, plus excess absorption at the source. Note the strong recovery at low energies.; (b) a partial covering model. The residuals left are at energies $\approx 8 \mathrm{keV} ;$ (c) additional high energy cutoff. Dots: SIS0, Circles: SIS1, Triangles: GIS2, Filled triangles: GIS3

Fig. 3.- Confidence contours of the power-law photon index against the absorbing column density (the photon index is $=1+\alpha$ ). Note the large best fit column density and the steep power-law spectrum. The horizontal lines represent the average spectral slope and range in $\mathrm{z} \approx 2$ radio-quiet quasars. The scale over which the plot is made shows $3 \sigma$ uncertainty in the previous observation. 


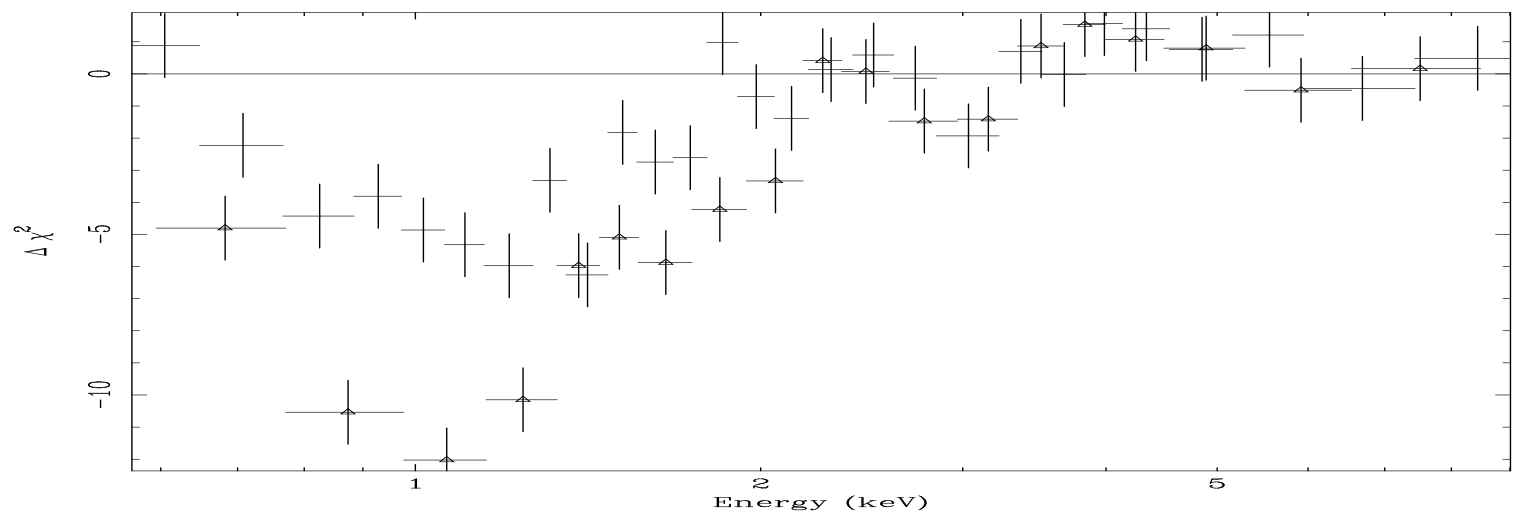



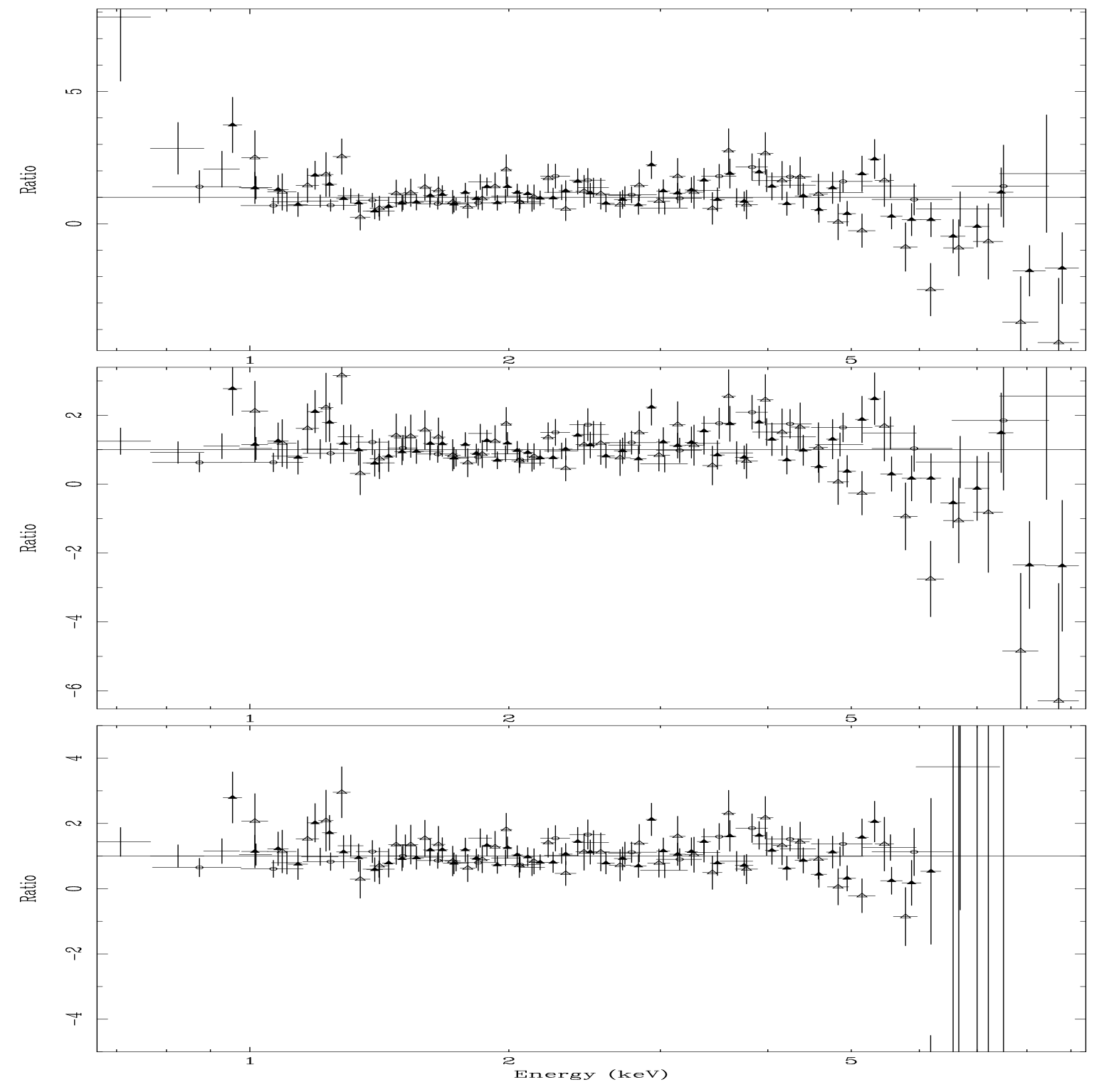


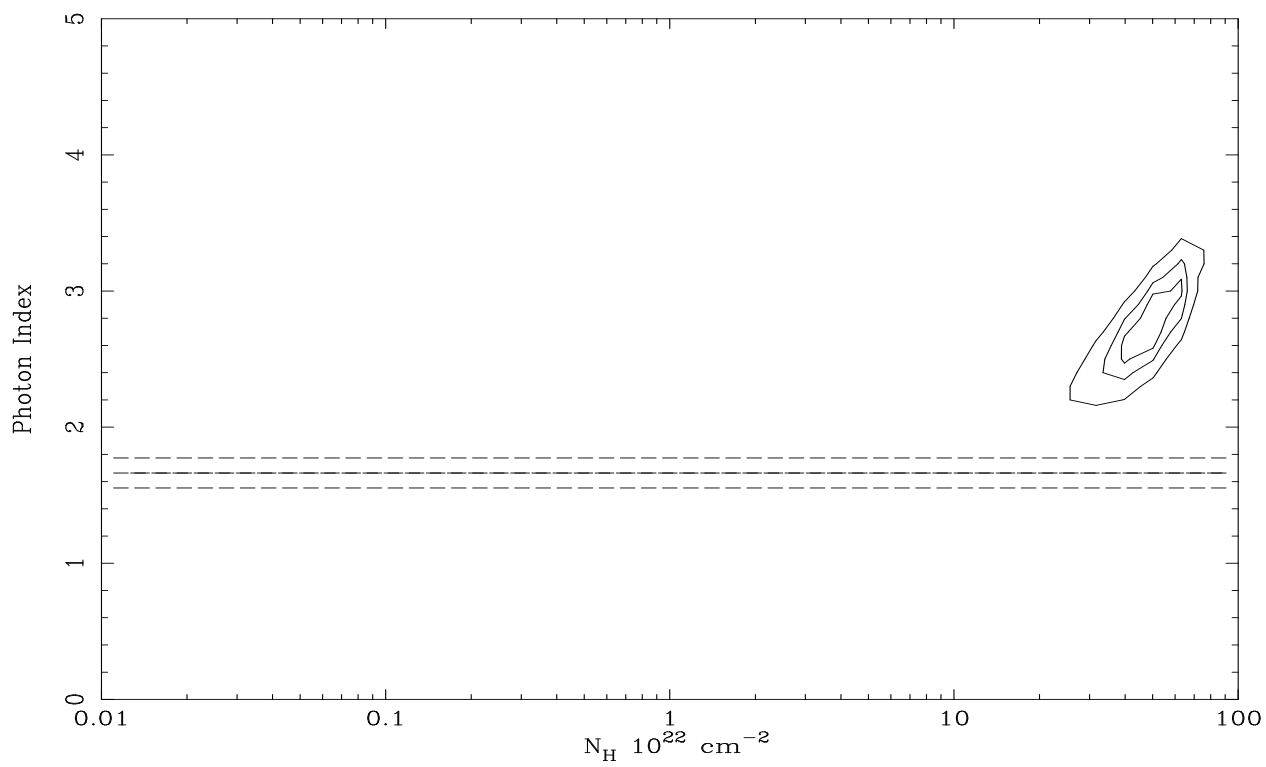

